\title{
PSEUDOLESÕES HEPÁTICAS NA TOMOGRAFIA COMPUTADORIZADA HELICOIDAL: ENSAIO ICONOGRÁFICO*
}

\author{
Gustavo de Souza Portes Meirelles ${ }^{1}$, Giuseppe D'Ippolito ${ }^{2}$
}

Resumo A tomografia computadorizada helicoidal é largamente empregada na avaliação do parênquima hepático e tem grande importância no planejamento clínico e cirúrgico. 0 fígado é o órgão que mais se beneficia de aquisições helicoidais, em fases diferentes da perfusão do parênquima, pela sua dupla vascularização e pela diferença de aporte sanguíneo entre tumores e parênquima sadio. Entretanto, várias armadilhas diagnósticas podem ser encontradas, dificultando a análise e prejudicando a diferenciação entre lesões verdadeiras e pseudolesões, principalmente aos olhos de radiologistas menos experientes. Essas pseudolesões têm forma, localização e características variadas, podendo simular lesões parenquimatosas. É de fundamental importância que estejamos aptos a reconhecê-las, no sentido de interpretar corretamente as imagens tomográficas. $O$ objetivo deste ensaio é classificar e ilustrar as diversas pseudolesões hepáticas pela tomografia computadorizada helicoidal, com uma breve descrição dessas lesões e com alternativas para diferenciá-las das lesões do parênquima.

Unitermos: Fígado; Tomografia computadorizada helicoidal; Erros de diagnóstico.

Abstract Helical computed tomography of hepatic pseudolesions: a pictorial essay.

Helical computed tomography has been widely used in the evaluation of the liver parenchyma for clinical and surgical management. The liver is probably the organ for which helical computed tomography examination is best indicated due to hepatic dual blood perfusion and the difference in blood supply between liver tumors and normal parenchyma. However, there are pitfalls in the interpretation of the images that may impair differentiation of true lesions from pseudolesions, particularly for the inexperienced radiologist. These pseudolesions present different shapes, location and characteristics that may mimic parenchymal lesions and their recognition and understanding are essential to avoid misdiagnoses. The aim of this study is to propose a systematic classification of the pseudolesions of the liver using illustrative cases and thus offer alternative means to differentiate these lesions from true parenchymal lesions.

Key words: Liver; Helical computed tomography; Pitfalls.

\section{INTRODUÇÃO}

Nos dias atuais, a tomografia computadorizada (TC) é um método rotineiro na avaliação do fígado, com ampla aceitação para a detecção e caracterização de tumores e com grande importância no planejamento clínico-cirúrgico e no prognóstico da doença ${ }^{(1)}$.

$\mathrm{O}$ advento dos aparelhos de TC helicoidais resultou em importante melhora na avaliação hepática, ao demonstrar, com maior eficácia, lesões parenquimatosas, o

\footnotetext{
* Trabalho realizado no Departamento de Diagnóstico po Imagem da Universidade Federal de São Paulo/Escola Paulista de Medicina (Unifesp/EPM), São Paulo, SP.

1. Pós-graduando do Departamento de Diagnóstico por Imagem da Unifesp/EPM.

2. Professor Adjunto, Chefe do Setor de Tomografia Computadorizada do Departamento de Diagnóstico por Imagem da Unifesp/EPM

Endereço para correspondência: Dr. Gustavo de Souza Por tes Meirelles. Rua Diogo de Faria, 650, apto. 33, Vila Clementino. São Paulo, SP, 04037-002. E-mail: gustavomeirelles@ terra.com.br

Recebido para publicação em 22/7/2002. Aceito, após revisão, em 19/2/2003.
}

que levou a uma maior utilização do método. No entanto, várias armadilhas diagnósticas surgiram, principalmente ligadas a defeitos de perfusão decorrentes da rápida injeção do meio de contraste e aquisição das imagens, dificultando a análise e prejudicando a diferenciação entre lesões e pseudolesões ${ }^{(1-4)}$.

Consideramos pseudolesões hepáticas aquelas imagens que simulam lesões hepáticas focais, podendo levar a diagnósticos equivocados. O objetivo deste estudo é apresentar uma revisão dessas pseudolesões, propondo uma classificação sistemática por meio de casos ilustrativos e estratégias para diferenciá-las de lesões parenquimatosas.

\section{CLASSIFICAÇÃO DAS PSEUDOLESÕES HEPÁTICAS}

Várias são as pseudolesões hepáticas encontradas na TC helicoidal. As principais encontram-se listadas no Quadro 1.
Quadro 1 Pseudolesões hapáticas na tomografia computadorizada helicoidal.

- Fissuras hepáticas acessórias
- Vasos não opacificados
- Efeito de volume parcial
- Alterações perfusionais focais
- Obstrução da veia cava superior
- Processos inflamatórios
- Irrigação venosa anômala
- Anastomoses arteriovenosas
- Tromboses portais
- Inversão do fluxo portal
- Compressões costais
- Neoplasias hipervasculares
- Alterações perfusionais difusas
- Esteatose focal
- Parênquima hepático preservado na estea-
tose difusa

\section{1 - Fissuras hepáticas acessórias}

Existem fissuras hepáticas incompletas, causadas por invaginações do diafragma, que podem subdividir o parênquima hepático (Figura 1). Encontram-se praticamente 
limitadas aos segmentos VII e VIII, próximas à cúpula diafragmática. Tornam-se mais proeminentes com a inspiração profunda e são mais comuns em pacientes enfisematosos e idosos ${ }^{(\mathbf{5})}$.

O conhecimento da sua morfologia e distribuição evita a maior parte dos erros diagnósticos. Pode-se ainda repetir o exame com inspiração superficial, o que promove atenuação ou até seu desaparecimento, ou usar reconstruções coronais e sagitais, que permitem definir a origem diafragmática da imagem ${ }^{(5,6)}$.

\section{2 - Vasos não opacificados}

Nas fases precoces de contrastação, veias hepáticas e ramos portais podem não ser opacificados, simulando lesões focais hipovasculares (Figura 2). Uma nova aquisição das imagens e a análise em conjunto das fases arterial e portal permitem solucionar o problema. Além disso, a diferenciação pode ser feita com a determinação da distribuição anatômica, trajeto e ramificações das estruturas venosas ${ }^{(3,7)}$.

\section{3 - Efeito de volume parcial}

As causas mais comuns são a flexura hepática, a cúpula diafragmática direita e a vesícula biliar (Figura 3). Lesões do pólo superior do rim direito, da adrenal direita e da porção descendente do duodeno podem ser outras causas de erro. O reconhecimento dessas pseudolesões é feito pela análise dos cortes adjacentes e, em casos de dúvidas, por meio de reconstruções coronais e sagitais ${ }^{(8)}$.

\section{4 - Alterações perfusionais hepáticas}

Podem ser focais ou difusas, por hiper ou hipoperfusão. Têm causas diversas, resultando de obstrução ao fluxo venoso sistêmico ou hepático, irrigação hepática anômala, variações anatômicas ou processos inflamatórios, alterações transitórias na perfusão hepática ou anastomoses arterioportais $^{(\mathbf{9 , 1 0})}$.

\section{1 - Alterações perfusionais focais por obstrução da veia cava superior}

Na oclusão da veia cava superior (VCS), o fluxo dos membros superiores segue vias acessórias, como o sistema ázigos-hemiázigos, o plexo venoso vertebral, as veias mamárias internas e torácicas laterais ${ }^{(\mathbf{1 1}, \mathbf{1 2})}$.

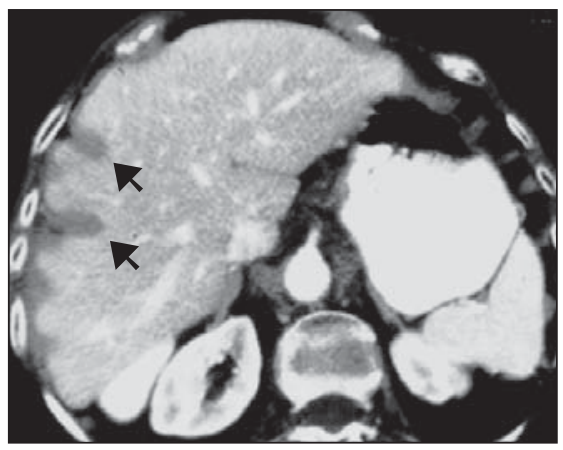

A

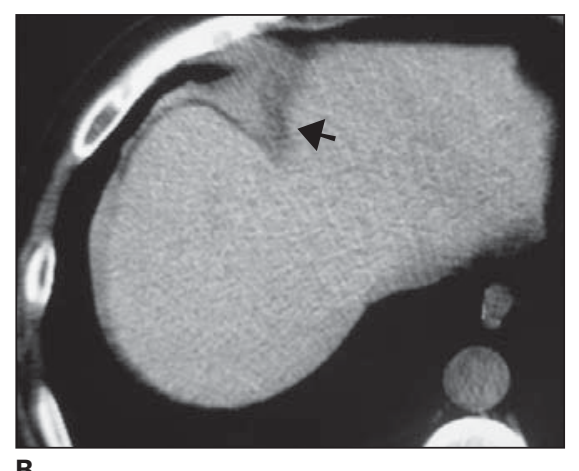

B

Figura 1. Fissuras hepáticas acessórias na tomografia computadorizada helicoidal. A: Endentações diafragmáticas na margem hepática lateral (setas). B: Fissura acessória na porção superior do fígado (seta).

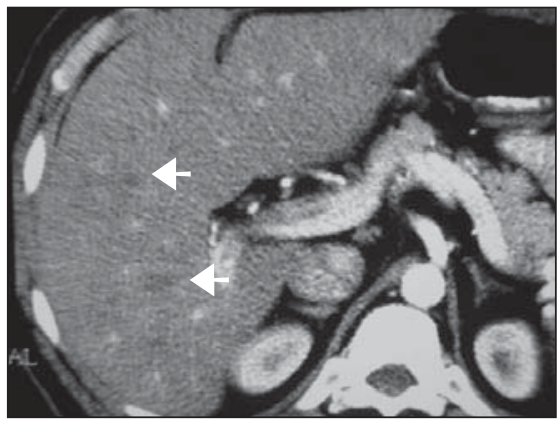

A

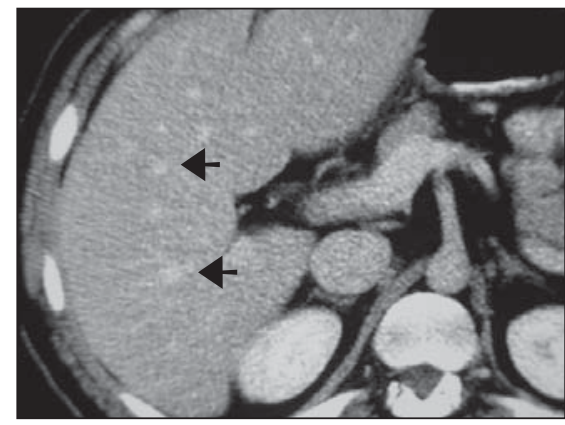

B

Figura 2. Pseudolesões causadas por veias hepáticas não contrastadas. A: Na fase arterial, ramos venosos não opacificados (setas) simulam lesões focais. B: Na fase portal há contrastação das veias hepáticas (setas), com realce homogêneo do parênquima.

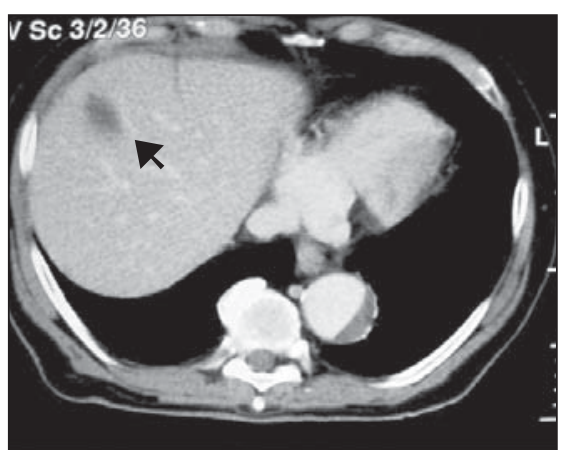

A

Figura 3. Pseudolesão por efeito de volume parcial da vesícula biliar. A: Imagem nodular hipoatenuante no lobo hepático direito (seta). B: Esta representa a cúpula da vesícula biliar (seta), bem visualizada na imagem adjacente.

Há contrastação precoce e dilatação dessas vias e realce intenso na fase arterial da porção anterior do segmento IV, que recebe sangue contrastado das colaterais (Figura 4). A diferenciação entre realce precoce e lesão parenquimatosa é possível pela análise da sua localização e aspecto característico, além da história de oclusão da VCS e da atenuação normal do segmento IV na fase portal ${ }^{(6,11)}$. O exame tomográ-
B

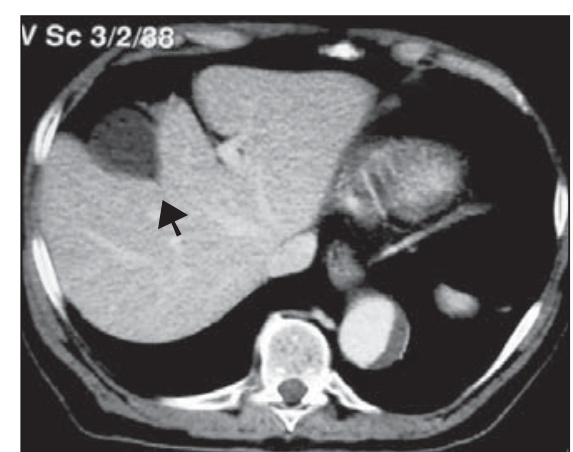

fico com contraste do tórax permite confirmar esta hipótese (Figura 4).

\section{2 - Alterações perfusionais focais por processos inflamatórios}

Áreas de realce hepático precoce e intenso podem ser evidenciadas em processos inflamatórios, principalmente na colecistite aguda (Figura 5), simulando o realce de lesões como hemangioma, hiperplasia 


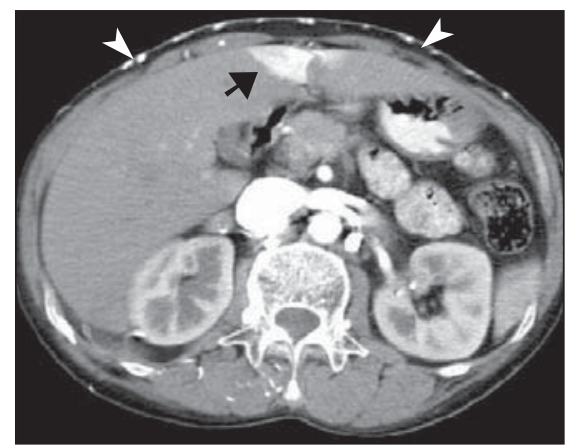

\section{A}

Figura 4. Trombose neoplásica da veia cava superior, causando alteração perfusional hepática. A: Área de hiperperfusão na fase arterial na porção anterior do segmento IV (seta), além de dilatação e realce intenso das veias subcutâneas (pontas de setas). B: Formação expansiva obstruindo a veia cava superior.

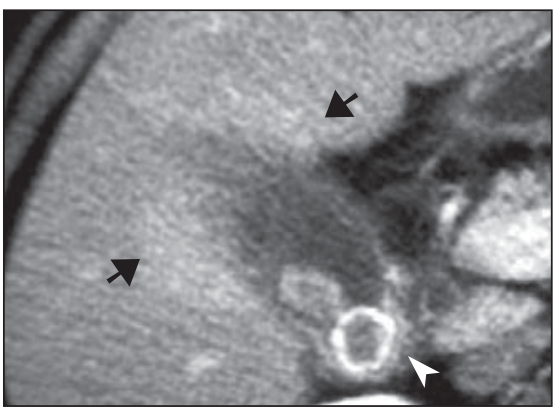

A

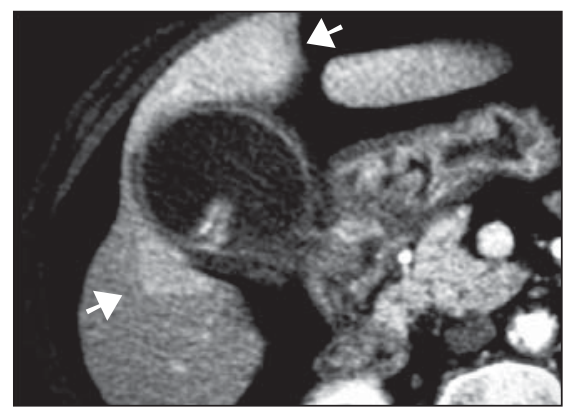

C

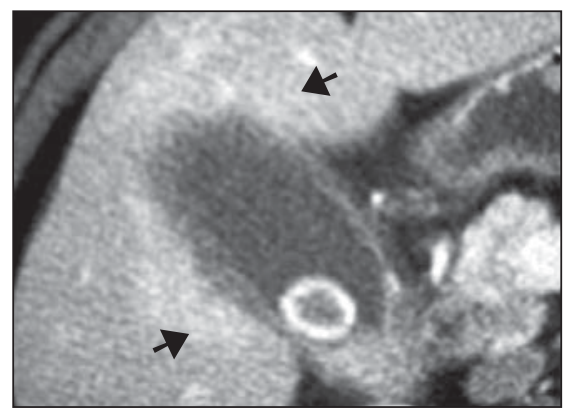

B

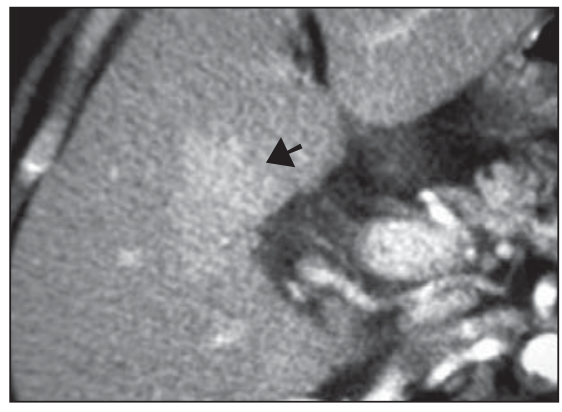

D

Figura 5. Alteração perfusional perivesicular na colecistite aguda. Cálculo impactado no infundíbulo da vesícula biliar (ponta de seta), com alteração de hiperperfusão no parênquima hepático adjacente (setas). nodular focal, metástases hipervasculares ou carcinoma hepatocelular ${ }^{(\mathbf{1 3})}$.

A diferenciação com lesões hepáticas primárias baseia-se na associação com o processo inflamatório, além do aspecto curvilíneo do realce e da localização perivesicular típica ${ }^{(\mathbf{1 3})}$. Deve-se lembrar ainda que nos cortes tardios a área de realce anômalo é normal ${ }^{(\mathbf{1 4})}$. É interessante observar que a área de parênquima hiperperfundido ao redor da vesícula biliar não traduz extensão do processo inflamatório da vesícula para o fígado.

\section{3 - Alterações perfusionais focais por retorno venoso anômalo}

$\mathrm{O}$ fígado pode ter uma vascularização suplementar por veias sistêmicas. Áreas irrigadas pelas vias suplementares apresentam realce intenso na fase arterial, com realce igual ou inferior ao do parênquima na fase portal ${ }^{(4,11)}$.

A alteração de perfusão na face posterior do segmento IV é uma das mais conhecidas (Figura 6), estando ligada a irrigação anômala através das veias gástricas, principalmente da direita ${ }^{(15)}$.

São comuns alterações perfusionais laterais ao ligamento falciforme (Figura 7), por irrigação aberrante pelas veias epigástricas e paraumbilical, exceto em cirróticos com fluxo hepatofugal, nos quais se deve sempre considerar a possibilidade de carcinoma hepatocelular ${ }^{(\mathbf{1 6 - 1 8})}$.

Alterações perfusionais podem também existir na fossa da vesícula biliar, por irrigação anômala a partir de veias císticas ${ }^{(\mathbf{1 5}}$ 17-19), e na região subcapsular, a partir de veias subcapsulares (Figura 8) $)^{(\mathbf{2 0 , 2 1})}$.

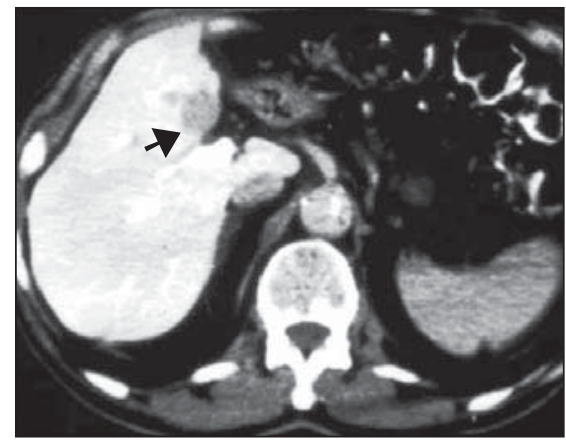

Figura 6. Pseudolesão hepática causada por alteração perfusional na face posterior do segmento IV. Portotomografia demonstrando área de hipoperfusão na face dorsal do segmento IV (seta) simulando lesão focal.

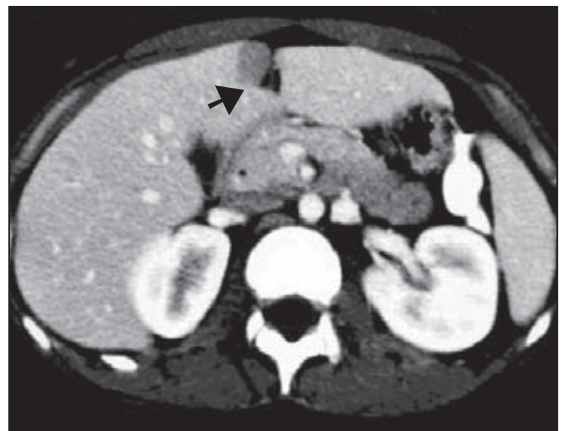

A

Figura 7. Alteração perfusional junto ao ligamento falciforme. A: Área focal de hipoperfusão na fase de contrastação portal, adjacente ao ligamento falciforme (seta). B: A tomografia computadorizada sem contraste venoso não demonstra alterações nesta localização, permitindo diferenciar este defeito de perfusão de esteatose focal. 
4.4 - Alterações perfusionais focais por anastomoses arteriovenosas

$\mathrm{Na}$ cirrose hepática, pequenas anastomoses arterioportais espontâneas podem causar pseudolesões centrais, com realce intenso na fase arterial (Figura 9). Nos cor-

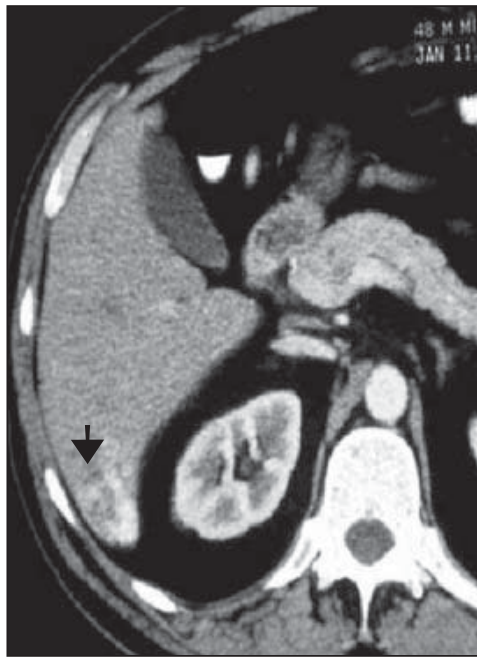

tes tardios as pseudolesões não devem aparecer, o que auxilia na diferenciação com o carcinoma hepatocelular ${ }^{(\mathbf{2 2 , 2 3})}$. Tumores hepáticos benignos e malignos (Figura 10), trauma e procedimentos invasivos podem causar anastomoses arterioportais ${ }^{(\mathbf{2 4})}$.

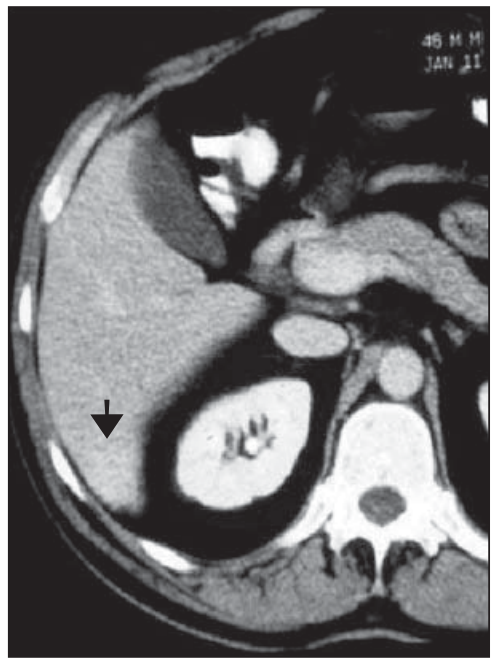

B
Figura 8. Pseudolesão hepática por alteração perfusional subcapsular. Realce hepático periférico (seta) nas fases de contrastação arterial (A) e portal (B), simulando lesão hipervascular.
4.5 - Alterações perfusionais focais por tromboses venosas

Tumores hepáticos malignos e, mais raramente, benignos podem ser causa de obstrução ao fluxo portal, com alterações de perfusão lobares, segmentares ou subsegmentares (Figura 11). Em alguns casos não se vê a lesão hepática focal, apenas a alteração perfusional (Figura 12) ${ }^{(\mathbf{3 , 4 , 1 1})}$.

4.6 - Alterações perfusionais focais por inversão do fluxo portal

Lesões expansivas podem comprimir o parênquima hepático, com inversão focal do fluxo portal e maior realce local na fase portal, muitas vezes interpretado como extensão da lesão (Figura 13). Essas alterações perfusionais são em banda, sem distribuição lobar ou segmentar, restritas ao local de compressão do parênquima ${ }^{(25)}$.

\section{7 - Outras alterações perfusionais} focais

Costelas muito arqueadas podem causar alterações perfusionais no local de compressão no parênquima hepático ${ }^{(\mathbf{1 9 , 2 6})}$.

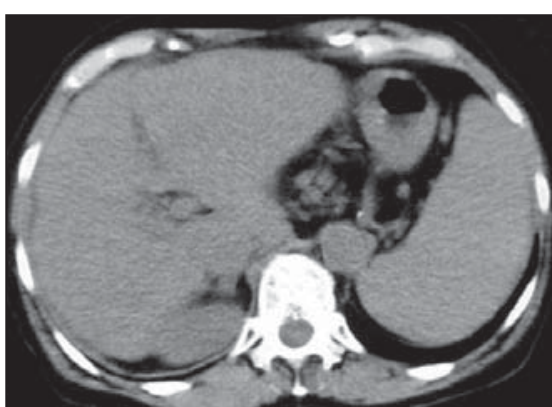

A

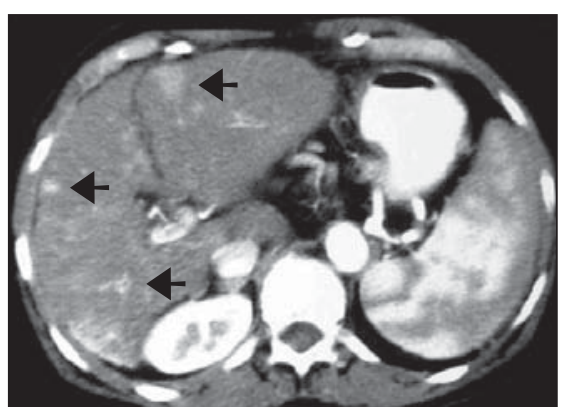

B

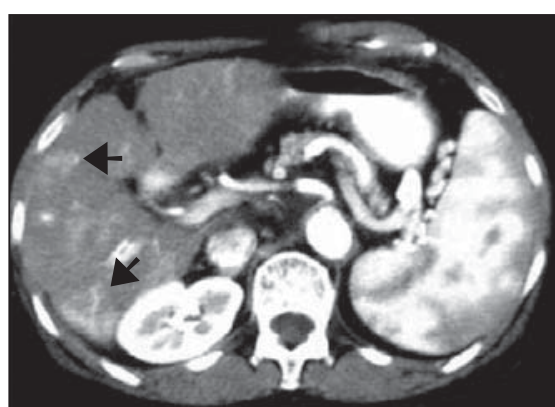

C

Figura 9. Pseudolesões hepáticas por anastomoses arteriovenosas espontâneas em paciente cirrótico. A: Tomografia computadorizada sem contraste intravenoso sem alterações hepáticas. B,C: Fase de contrastação arterial evidenciando múltiplas áreas de hiperperfusão (setas) no parênquima hepático.

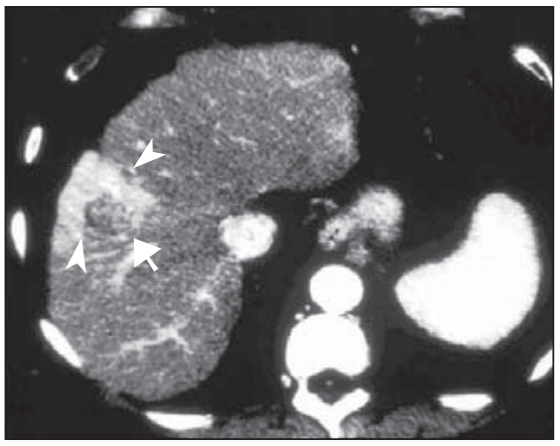

Figura 10. Anastomoses arteriovenosas por hepatocarcinoma. As fases arterial (A) e portal (B) de contrastação hepática demonstrando imagem nodular (setas) com realce parenquimatoso adjacente (pontas de setas).

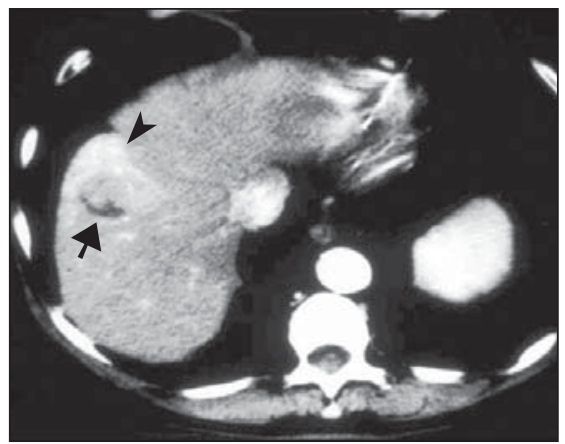

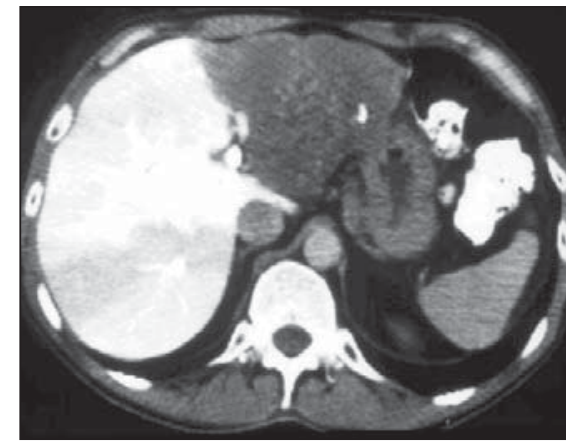

Figura 11. Pseudolesão por alteração perfusiona focal decorrente de trombose portal. Portotomografia evidenciando metástase no lobo esquerdo com obstrução portal e hiperperfusão no lobo direito. 


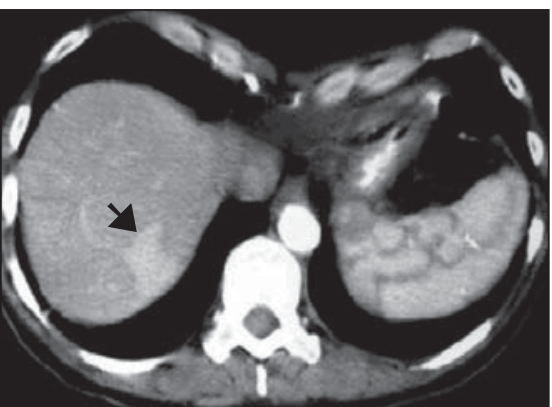

A

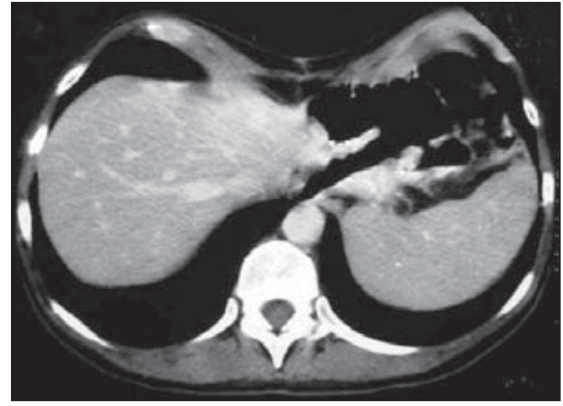

C

Figura 12. Alteração perfusional hepática segmentar. Fases de contrastação arterial (A) e portal (B) demonstrando área de hiperperfusão no lobo direito (setas), não se caracterizando lesão focal. Nas fases tardia (C) e sem contraste venoso (D) não se observam alterações hepáticas.

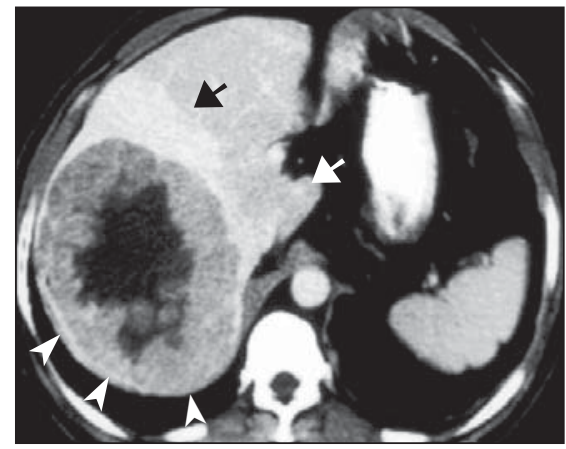

A

Figura 13. Alteração perfusional focal por inversão do fluxo portal. A: Parênquima hepático comprimido por metástase (pontas de setas), com alteração focal de hiperperfusão (setas). B: Alteração perfusional (setas) por inversão do fluxo portal devido a colangiocarcinoma (pontas de setas).

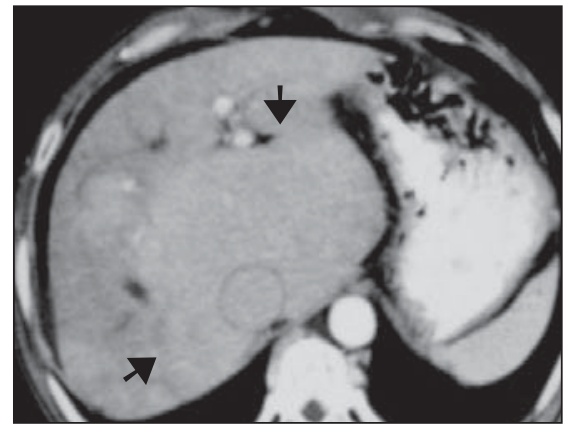

A

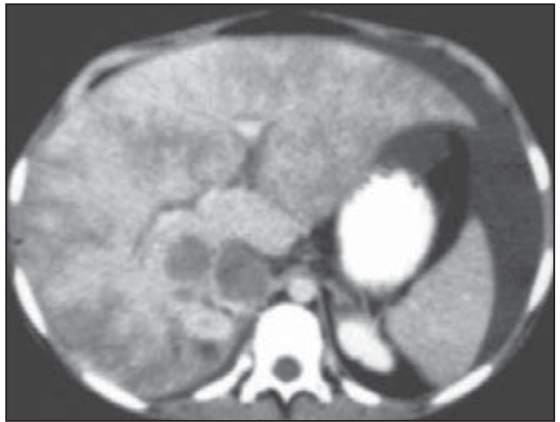

B

Figura 14. Síndrome de Budd-Chiari com alteração perfusional hepática difusa. A: Trombose das veias hepáticas com hipertrofia do lobo caudado (setas). B: Realce heterogêneo do parênquima hepático, por inversão do fluxo portal.
Neoplasias hipervasculares podem determinar hipoperfusão parenquimatosa adjacente ${ }^{(4,11)}$.

\section{5 - Alterações perfusionais difusas}

Algumas condições clínicas podem provocar alterações de perfusão difusas, entre elas a cirrose, a síndrome de BuddChiari, a insuficiência cardíaca congestiva, a pericardite constritiva e a fibrose mediastinal ${ }^{(4,9,11,14)}$

A síndrome de Budd-Chiari é uma das causas mais conhecidas de alterações perfusionais difusas, promovendo inversão do fluxo portal e realce heterogêneo do parênquima hepático (Figura 14) ${ }^{(11)}$

\section{6 - Esteatose focal}

Geralmente difusa, mas ocasionalmente focal, é uma das causas mais freqüentes de pseudolesão hepática (Figura 15). Suas localizações mais típicas são as adjacências do ligamento falciforme, regiões subcapsulares, perivesiculares, lobo caudado e porção posterior do segmento $\mathrm{IV}^{(27,28)}$.

O depósito de gordura pode ser rapidamente reversível e transitório, desaparecendo em exames posteriores, principalmente se afastado o fator clínico causador da esteatose (Figura 16).

Quando a esteatose é geográfica (Figura 17) ou multinodular (Figura 18), o diagnóstico diferencial inclui o carcinoma hepatocelular, abscessos, metástases e hemangiomas ${ }^{(27,28)}$

A diferenciação entre esteatose e lesão neoplásica é de grande importância. A esteatose não tem efeito expansivo, tem atenuação muito baixa e geralmente apresenta vasos hepáticos e portais não distorcidos

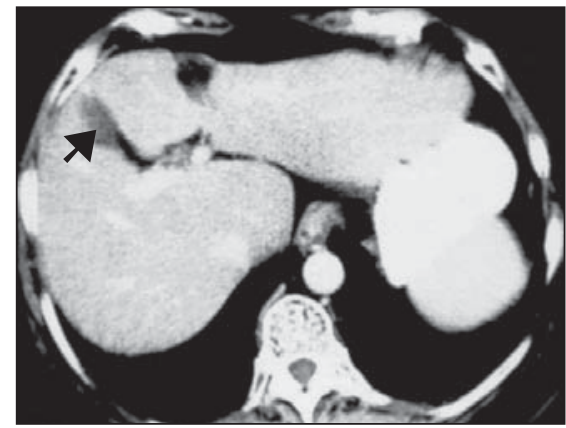

Figura 15. Esteatose hepática focal (seta) na tomografia computadorizada helicoidal. Nota-se ainda um cisto no lobo esquerdo. 


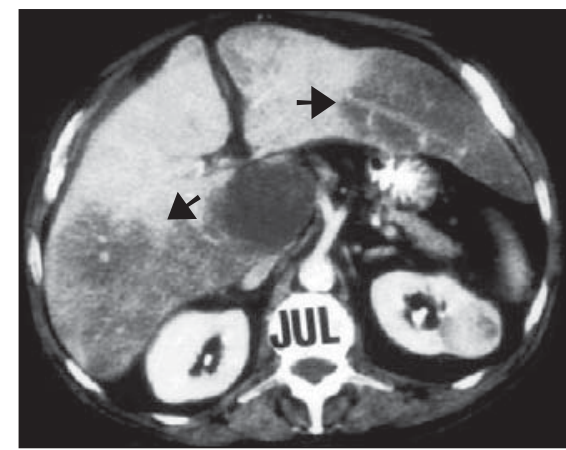

A

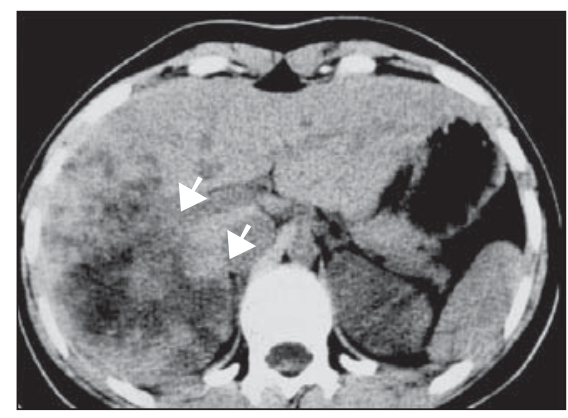

A

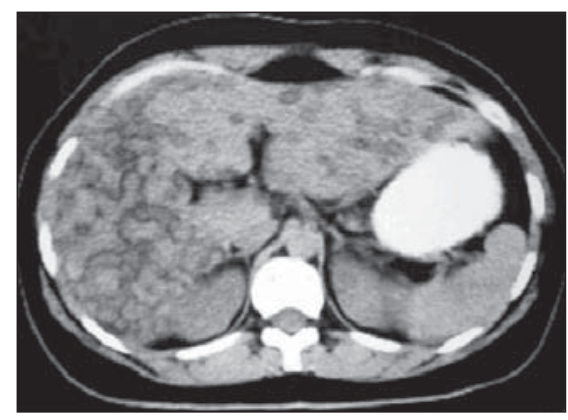

A

no seu interior, ao contrário de lesões do parênquima. Porém, é importante observar que, eventualmente, metástases hepáticas confluentes podem envolver vasos hepáticos sem distorcê-los. A ressonância magnética tem grande valor no diagnóstico da esteatose, utilizando-se sequiências que suprimem o sinal da gordura, como a gradiente-eco em fase e oposição de fase ${ }^{(\mathbf{2 9})}$.

\section{7 - Parênquima hepático preservado na esteatose difusa}

Os locais mais comuns são a região perivesicular (Figura 19), subcapsular, o lobo caudado (Figura 20) e a porção medial inferior do fígado.

O parênquima preservado tem atenuação superior à do fígado esteatótico (Figura

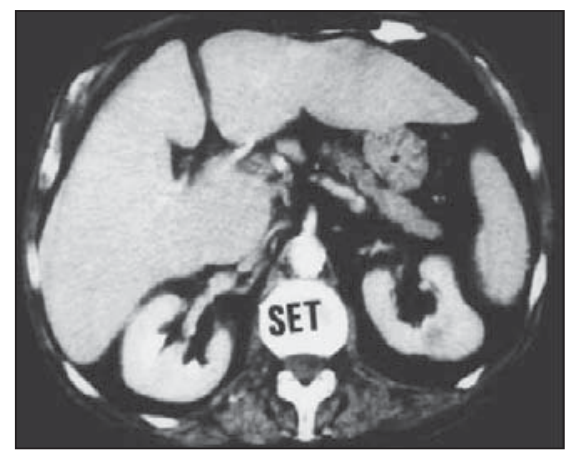

B

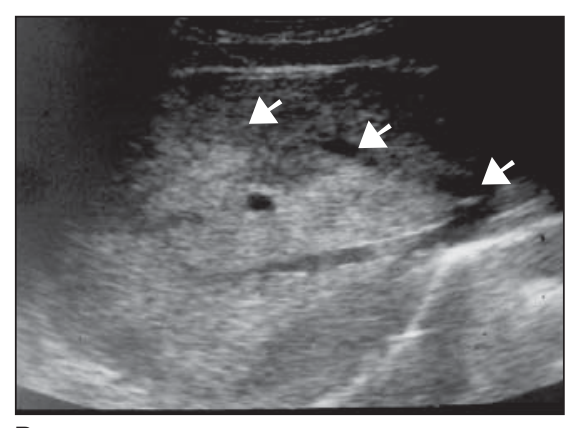

B

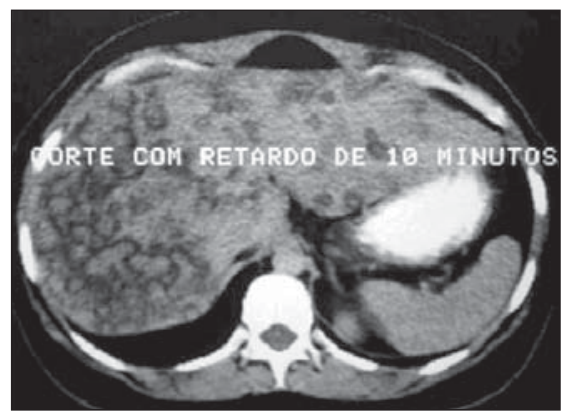

B

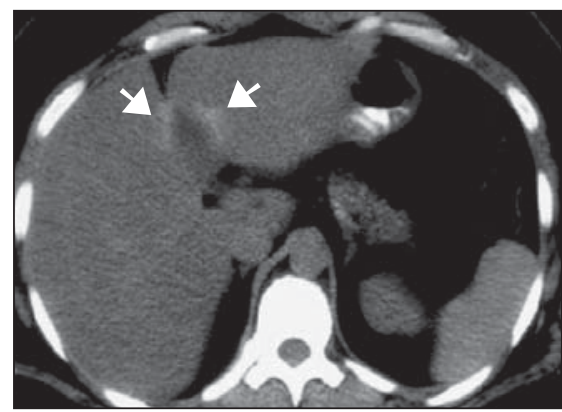

Figura 19. Áreas de parênquima hepático preservado na região perivesicular (setas).

21). A diferenciação com tumores se faz por sua atenuação, localização e morfologia. Nos casos duvidosos pode-se recorrer à cintilografia, à ressonância magnética e, caso necessário, à biópsia ${ }^{(6,8,30,31)}$.
Figura 16. Esteatose hepática segmentar, com resolução completa em exame posterior, realizado após dois meses. A: Áreas segmentares de esteatose hepática (setas). B: Desaparecimento dessas áreas na tomografia computadorizada de controle. Como achado incidental, nota-se nódulo sólido no rim esquerdo.

Figura 17. Esteatose hepática geográfica. A: Imagem hipoatenuante no lobo hepático direito (setas), com limites mal definidos, simulando lesão secundária. B: Ultra-sonografia mostrando imagem hiperecogênica nessa topografia, com vasos no interior não distorcidos e sem efeito expansivo, compatível com esteatose (setas).

Figura 18. Esteatose hepática multinodular, simulando metástases. Múltiplas áreas hipoatenuantes distribuídas ao longo do leito portal e difusamente pelo parênquima hepático, sem efeito expansivo.

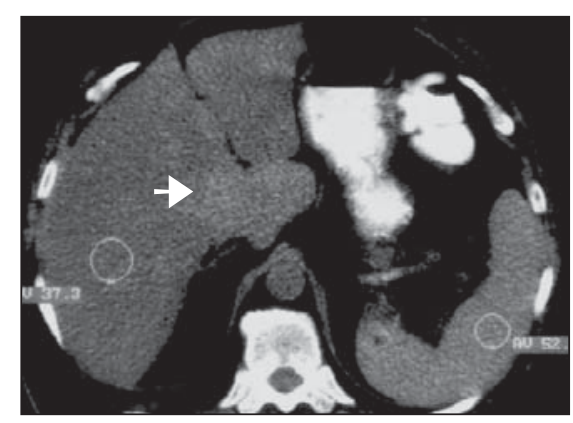

Figura 20. Parênquima hepático preservado no lobo caudado (seta) em fígado esteatótico.

\section{CONCLUSÃO}

As pseudolesões hepáticas são comuns na TC helicoidal e provavelmente aumentarão em incidência com a maior utilização 


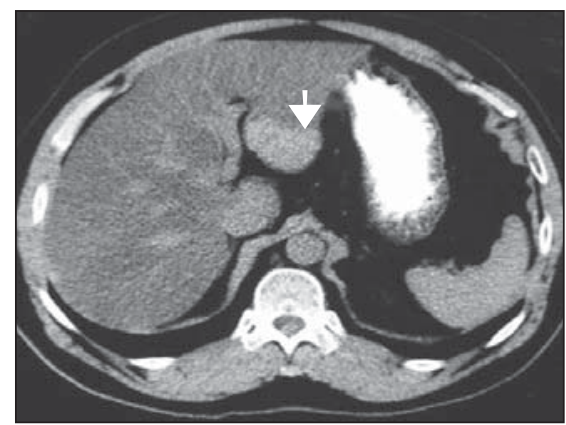

Figura 21. Área de parênquima preservado no lobo hepático esquerdo (seta).

dos tomógrafos "multi-slice". Em geral são facilmente distinguíveis de lesões hepáticas, sendo necessário estarmos habituados a reconhecê-las.

A compreensão da etiologia dessas pseudolesões e o conhecimento do seu aspecto tomográfico são os primeiros passos para diferenciá-las de lesões verdadeiras e elevar a eficácia do método.

\section{REFERÊNCIAS}

1. Urban BA, McGhie PA, Fishman EK. Helical CT: diagnostic pitfalls of arterial phase imaging of the upper abdomen. AJR 2000;174:455-61.

2. Silverman PM, Cooper CJ, Weltman DI, Zeman RK. Helical CT: practical considerations and potential pitfalls. RadioGraphics 1995;15:25-36.

3. Oliver JH 3rd, Baron RL. Helical biphasic contrastenhanced CT of the liver: technique, indications interpretation, and pitfalls. Radiology 1996;201:114.

4. Chen WP, Chen JH, Hwang JI, et al. Spectrum of transient hepatic attenuation differences in biphasic helical CT. AJR 1999;172:419-24.

5. Auh YH, Rubenstein WA, Zirinsky K, et al. Accessory fissures of the liver: CT and sonographic appearance. AJR 1984;143:565-72.
6. Shirkhoda A. Diagnostic pitfalls in abdominal CT. RadioGraphics 1991;11:969-1002.

7. Herts BR, Einstein DM, Paushter DM. Spiral CT of the abdomen: artifacts and potential pitfalls. AJR 1993;161:1185-90.

8. Mirowitz SA. Liver and biliary system. In: Mirowitz SA, ed. Pitfalls, variants and artifacts in body MR imaging. St. Louis, MO: Mosby-Year Book, 1996.

9. Galvão Filho MM. Alterações de perfusão nos exames de portotomografia: características e frequiência. (Tese de Doutorado). São Paulo: Universidade Federal de São Paulo, 1998.

10. Nelson RC, Thompson GH, Chezmar JL, Harned RK 2nd, Fernandez MP. CT during arterial portography: diagnostic pitfalls. RadioGraphics 1992; 12:705-20.

11. Gryspeerdt S, Van Hoe L, Marchal G, Baert AL. Evaluation of hepatic perfusion disorders with double-phase spiral CT. RadioGraphics 1997;17: 337-48.

12. Trigaux J, Lacrosse M, Daube A. Venous return by the paraumbilical and hepatic veins in case of superior vena cava obstruction. Abdom Imaging 1996;21:504-6.

13. Yamashita K, Jin MJ, Hirose Y, et al. CT finding of transient focal increased attenuation of the liver adjacent to the gallbladder in acute cholecystitis. AJR 1995;164:343-6.

14. Ito K, Mitchell DG, Honjo K, et al. Biphasic contrast-enhanced multisection dynamic MR imaging of the liver: potential pitfalls. RadioGraphics 1997; 17:693-705.

15. Matsui O, Takahashi S, Kadoya M, et al. Pseudolesion in segment IV of the liver at CT during arterial portography: correlation with aberrant gastric venous drainage. Radiology 1994;193:31-5.

16. Ohashi I, Ina H, Gomi N, et al. Hepatic pseudolesion in the left lobe around the falciform ligament at helical CT. Radiology 1995;196:245-9.

17. Nelson RC, McDermott VG, Paulson EK. Aberrant venous drainage to the liver: imaging implications. Radiology 1995;197:338-40.

18. Hiwatashi A, Yoshimitsu K, Honda H, et al. Pseudolesion in segment II of the liver observed on CT during arterial portography caused by the aberrant left gastric venous drainage. Abdom Imaging 1999; 24:357-9

19. Bluemke DA, Soyer P, Fishman EK. Nontumorous low-attenuation defects in the liver on helical CT during arterial portography: frequency, location, and appearance. AJR 1995;164:1141-5.

20. Llauger J, Perez C, Pallardo Y, Zidan A. Perihiliar low density areas: a pitfall in CT-portography. Eur J Radiol 1992;14:11-2.

21. Soyer P, Lacheheb D, Levesque M. False-positive CT portography: correlation with pathologic findings. AJR 1993;160:285-9.

22. Kim TK, Choi BI, Han JK, Chung JW, Park JH, Han MC. Nontumorous arterioportal shunt mimicking hypervascular tumor in cirrhotic liver: two-phase spiral CT findings. Radiology 1998;208:597-603.

23. Yu JS, Kim KW, Sung KB, Lee JT, Yoo HS. Small arterial-portal venous shunts: a cause of pseudolesions at hepatic imaging. Radiology 1997;203 737-42.

24. Ito K, Honjo K, Fujita T, Awaya H, Matsumoto T, Matsunaga N. Enhanced MR imaging of the liver after ethanol treatment of hepatocellular carcinoma: evaluation of areas of hyperperfusion adjacent to the tumor. AJR 1995;164:1413-7.

25. Mathieu D, Luciani A, Achab A, Zegai B, Bouanane M, Kobeiter H. Hepatic pseudolesions. Gastroenterol Clin Biol 2001;25(4 Suppl):B158-66.

26. Kanematsu M, Kondo H, Enya M, Yokoyama R, Hoshi H. Nondiseased portal perfusion defects adjacent to the right ribs shown on helical CT during arterial portography. AJR 1998;171:445-8.

27. Paulson EK, Baker ME, Spritzer CE, Leder RA, Gulliver DJ, Meyers WC. Focal fatty infiltration: a cause of nontumorous defects in the left hepatic lobe during CT arterial portography. J Comput Assist Tomogr 1993;17:590-5.

28. Yoshikawa J, Matsui O, Takashima T, et al. Focal fatty change of the liver adjacent to the falciform ligament: CT and sonographic findings in five surgically confirmed cases. AJR 1987;149:491-4.

29. Wenker JC, Baker MK, Ellis JH, Glant MD. Focal fatty infiltration of the liver: demonstration by magnetic resonance imaging. AJR 1984;143:573-4.

30. Rocha MS, Cerri GG, Rocha DC, Magalhães A. Área hipoecogênica ultra-sonográfica periportal: importante pseudolesão hepática. Radiol Bras 1988;21:181-4.

31. Scriven MW, Shandall A, Fitzgerald EJ, Puntis MC. Hepatic 'pseudotumours': an important diagnostic pitfall. Ann R Coll Surg Engl 1993;75:43-5 